\title{
Synthesis of Si and CdTe quantum dots and their combined use as down-shifting photoluminescent centers in Si solar cells
}

\author{
R. Guerrero-Gonzalez ${ }^{1,2} \cdot$ F. A. Orona ${ }^{1,2} \cdot$ E. Saucedo-Flores ${ }^{2} \cdot$ R. Ruelas $^{2}\left(\right.$ D $\cdot$ J. E. Pelayo-Ceja ${ }^{1,2,3}$ (D) \\ R. Lopez-Delgado ${ }^{3}$ A. Cordova-Rubio ${ }^{1,3} \cdot$ M. E. Álvarez-Ramos ${ }^{3} \cdot$ A. Ayon $^{1}$
}

Received: 19 September 2018 / Accepted: 13 August 2019 / Published online: 21 August 2019

(c) The Author(s) 2019

\begin{abstract}
This paper describes the synthesis and characterization of Si and CdTe quantum dots (QDs) and their use, either on their own or combined, as photoluminescent (PL) down-shifting nanostructured coatings aimed to enhance the photovoltaic efficiency of polycrystalline silicon solar cells. To this end, the front face of a set of silicon cells was coated with different volume ratios of the above-mentioned QDs, or some of its mixtures, dispersed in PMMA layers. Previously, the absorption and the PL $(\mathrm{exc}=380 \mathrm{~nm})$ response of the dispersions of the QDs were measured. It was observed that the PL response of the mixtures was strongly affected in location, spread, and intensity of the emission peak according to the volume ratio involved. As compared to the unmixed CdTe samples, a notorious red-shift of the main peak location was obtained for a couple of mixed QDs' dispersions, which was one of the project objectives given that Si solar cells respond better to photons with wavelengths in the 650-700 nm range. This effect was confirmed in a set of polycrystalline Si solar cells covered with and without nanostructured PMMA/QDs layers tested under AM 1.5G solar simulator conditions. It was found that the use of the proposed mixtures of QDs gave an increase of $1.53 \%$ in solar cell power conversion efficiency.
\end{abstract}

Keywords Silicon solar cells · Cadmium telluride $\cdot$ Quantum dots · Down-shifting

\section{Introduction}

The total solar energy density outside the atmosphere, known as the irradiance solar constant at AM0 conditions, is $I_{\mathrm{SC}}=1367 \mathrm{Watt} / \mathrm{m}^{2}$; and approximately, $98 \%$ of the power density in the solar spectrum lays in the $200-2500 \mathrm{~nm}$ wavelength range, that is, from the near UV to the medium near IR and has a modal peak at about $500 \mathrm{~nm}$. It has been reported that, due to fundamental losses, the maximum efficiency theoretically achievable in a Si solar cell is of $30.1 \%$

\section{J. E. Pelayo-Ceja}

pcje@hotmail.es

1 MEMS Research Lab, Department of Physics and Astronomy, University of Texas at San Antonio, One UTSA Circle, San Antonio, TX 78249, USA

2 Centro de Ciencias Exactas e Ingenierías, Universidad de Guadalajara, Blvd. Gral. Marcelino García Barragán 1421, Olímpica, 44430 Guadalajara, Jal., Mexico

3 Departamento de Física, Universidad de Sonora, Blvd. Luis Encinas y Rosales, Col. Centro, 83000 Hermosillo, Sonora, Mexico
[1]. Intrinsic and extrinsic losses account for the energy unaccounted for. Two of these intrinsic losses, related to the Si band-gap energy $E_{\mathrm{G}}$, are depicted in Fig. 1 for two segments of the solar spectrum, namely, for the wavelength ranges of $\lambda \leq 1107 \mathrm{~nm}$ and $\lambda>1107 \mathrm{~nm}$, or equivalently, for photon energy values $E_{\mathrm{ph}} \geq E_{\mathrm{G}}$ and $E_{\mathrm{ph}}<E_{\mathrm{G}} \mathrm{eV}$, knowing that $E_{\mathrm{G}}=1.12 \mathrm{eV}$. For the latter range, photons are not absorbed in $\mathrm{Si}$ and, therefore, their energy is not usable. This is indicated by the parameter $I_{\mathrm{NA}}(\lambda)$ in Fig. 1 which corresponds to an $E_{\mathrm{ph}}(\lambda)$ loss of approximately $20 \%$ of the incident $I_{\mathrm{SC}}$, represented by the shaded area under $E_{\mathrm{G}}$ in the same Fig. 1 . Regarding the $E_{\mathrm{ph}} \geq E_{\mathrm{G}}$ range, absorbed photons are sufficiently energetic to generate electron-hole $(e-h)$ pairs; however, the energy in excess of $E_{\mathrm{G}}$ is lost by carrier collisions with the semiconductor lattice (phonon creation) and, consequently, generate heat. Assuming that all the energy in excess of $E_{\mathrm{G}}$ goes to phonon creation, the energy loss term can then be defined as follows:

$I_{Q}=100\left(\frac{E_{\mathrm{ph}}(\lambda)-E_{\mathrm{G}}}{E_{\mathrm{ph}}(\lambda)}\right)(\%)$ for $\lambda \leq 1107 \mathrm{~nm}$, 


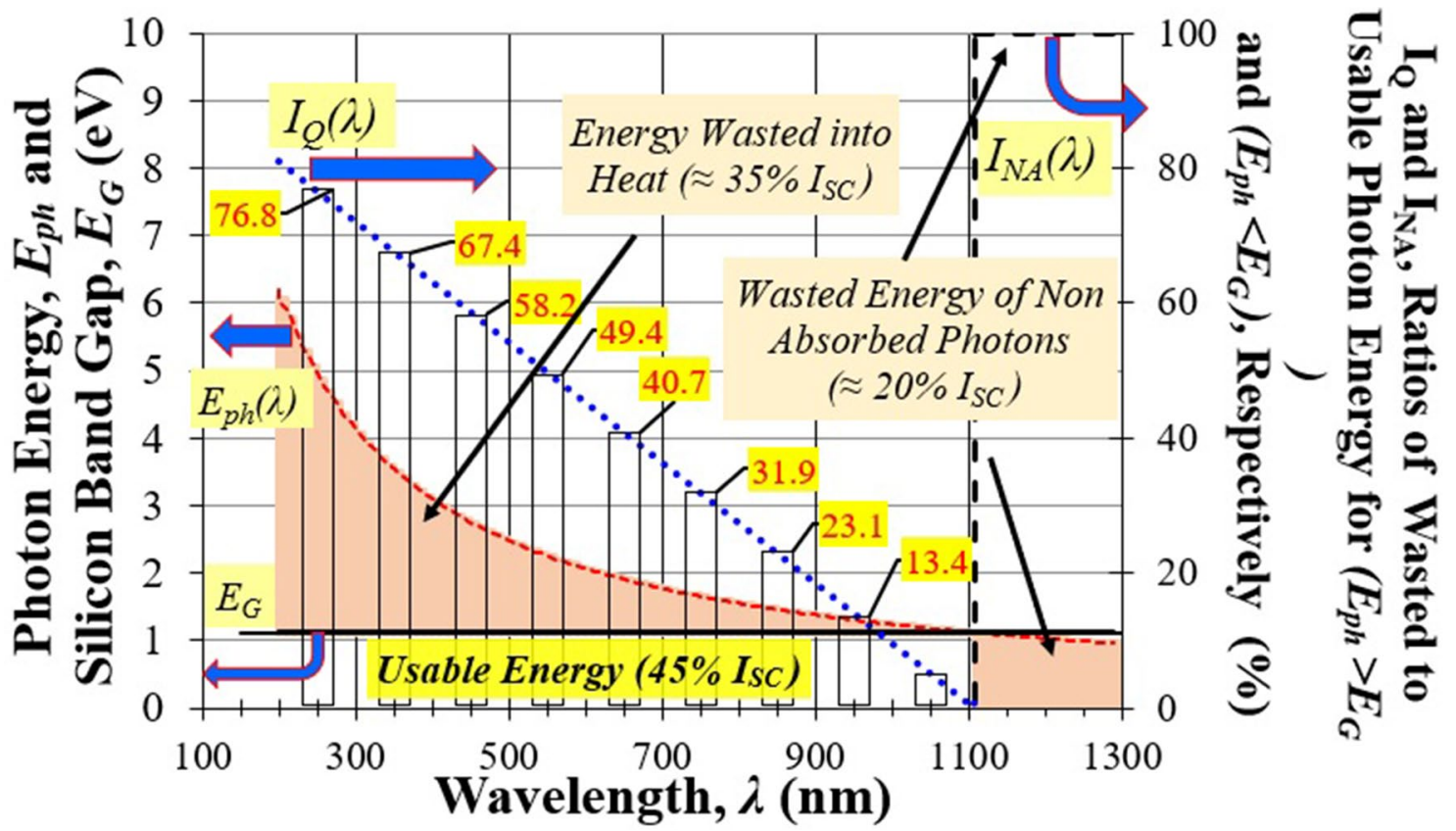

Fig. 1 Distribution of the spectral fundamental losses in $\mathrm{Si}$

which describes how the ratio of the areas between $E_{\mathrm{ph}}$ and $E_{\mathrm{G}}$ varies along the $E_{\mathrm{ph}} \geq E_{\mathrm{G}}$ wavelength range. The loss fraction corresponding to the above expression, indicated by the shaded area over $E_{\mathrm{G}}$ in Fig. 1, integrates to $35 \%$ of $I_{\mathrm{SC}}$, indicating that the usable energy reduces to approximately $45 \%$ of $I_{\mathrm{SC}}$ in the aforementioned wavelength range. Ostensibly, the larger the energy of the photon absorbed, the larger the portion of energy lost. This is indicated in Fig. 1 by the $I_{Q}$ parameter associated with the segmented means for different photon energies, parameter that increases from a relatively small loss of $I_{Q}=13.4 \%$ for wavelengths of $\sim 950 \mathrm{~nm}$, for example, to $I_{Q}=76.8 \%$ for a photon wavelength of $\sim 257 \mathrm{~nm}$. Ostensibly, a Si cell response for highenergy photons (smaller wavelengths) is rather modest. A concomitant effect of the heat loss is an increase of the solar cell temperature and of its leakage current, resulting in degradation of the device fill factor $\mathrm{I}-\mathrm{V}$ response and of its power conversion efficiency.

Si solar cells are also affected by other losses mainly related to carrier low lifetime and/or short diffusion length which imply increased $e-h$ pair recombination loss rate [2]. This loss is monitored in solar cells by measuring their external quantum efficiency (EQE), which provides the spectral information regarding how close a single absorbed photon is of producing an effective circuit current of one electron. Furthermore, the 1-EQE parameter defines the $e-h$ pair recombination per absorbed photon. Figure 2 provides an example for a typical EQE plot for a Si solar cell; the 1-EQE trace is also added along with a segmented distribution of it. Ostensibly, the absorption of short-wavelength photons (i.e., UV range) in Si solar cells is far from efficient. Specifically, in the $200-400 \mathrm{~nm}$ wavelength range, $1-\mathrm{EQE} \approx 2 / 3$, indicating that an average of around three absorbed photons are needed to produce an effective current of one electron, and thus, the recombination of two $e-h$ pairs takes place.

Several technical approaches, mostly related to emitter diffusion or front surface passivation optimization, have been disclosed aimed at mitigating the above-mentioned $\mathrm{Si}$ solar cells' UV-response limitations [3-5]. The method discussed in this paper is based on employing the down-shifting photoluminescent (DSPL) properties observed in QDs [6, 7], which are capable of absorbing UV photons and subsequently emitting lower energy photons that have a better match for Si solar cells [8]. Si and CdTe QDs were selected for achieving this goal, incorporating them into a PMMA [9] matrix applied as a DSPL coating layer on polycrystalline Si solar cells.

Specifically, the window surface of a set of silicon cells was coated with different volume ratios of the above-mentioned QDs dispersed in PMMA layers. Previously, the absorption and the PL response of several dispersions with varying quantities and proportions of QDs were determined using UV excitation with a wavelength of $380 \mathrm{~nm}$. It was observed that the PL response of the mixtures was strongly affected in location, spread, and intensity of the emission peak according to the volume ratio involved. Specifically, compared to the unmixed CdTe QD samples, a blue-shift of the main CdTe peak location was observed in SiQD-rich dispersions and a red-shift in CdTe-rich dispersions that could help increase the efficiency of $\mathrm{Si}$ solar cells considering 


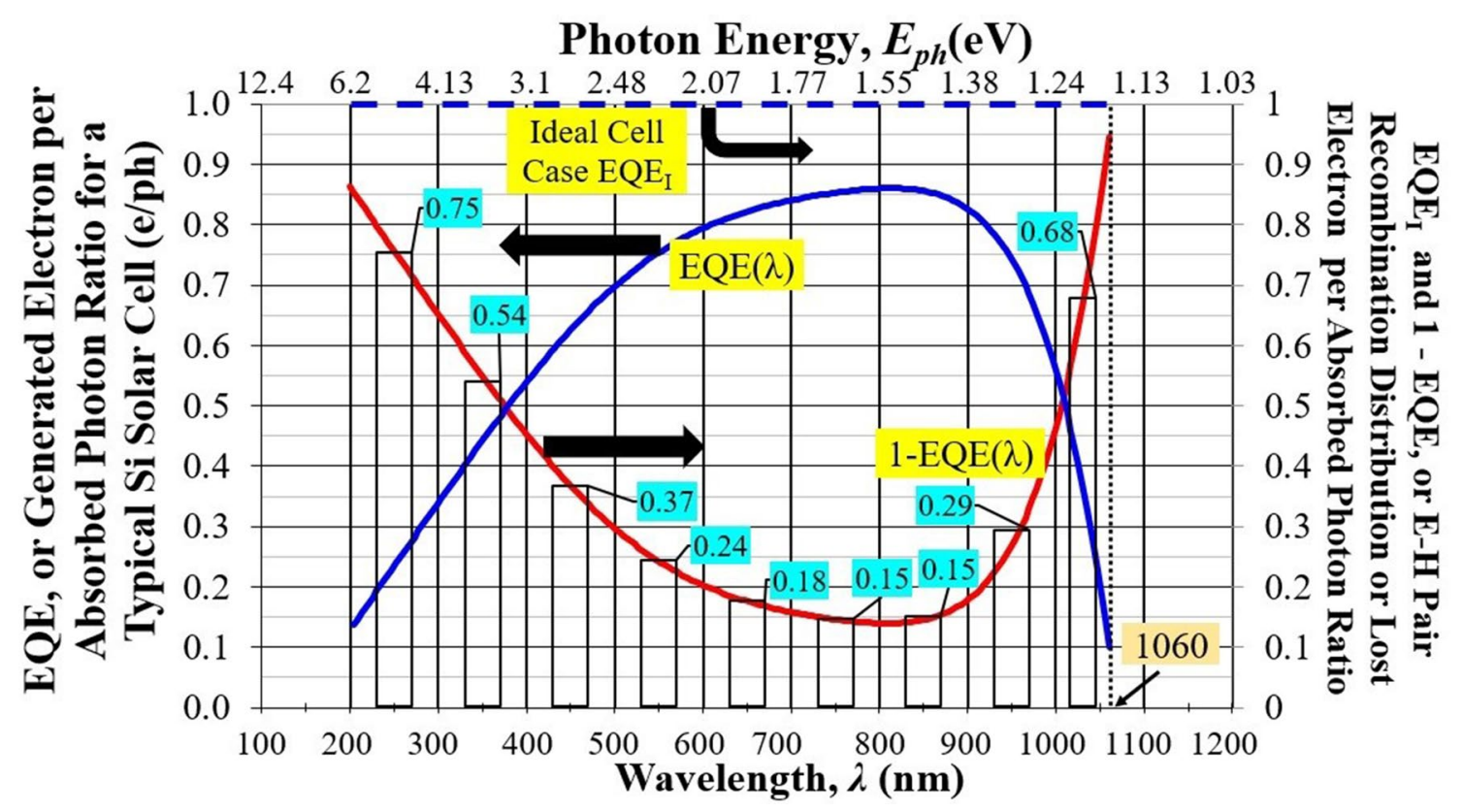

Fig. 2 EQE and carrier recombination loss distribution (1-EQE)

that they respond better to photons with wavelengths in the $650-700 \mathrm{~nm}$ range. This effect was confirmed in a set of polycrystalline Si solar cells coated with nanostructured PMMA layers, tested under AM 1.5G solar simulator conditions, and compared to the previous test results of the bare solar cells.

\section{Experimental details}

\section{Synthesis of Si quantum dots}

The silicon QDs' solutions were synthesized at room temperature [10] in a water-based dispersion, following the method reported by Wang et al. [11]. The process was observed to be relatively straightforward and produced consistent results. The synthesis procedure can be briefly described as follows: (1) initiate with $4 \mathrm{ml}$ of deionized water (DI) in a vial; (2) dropwise addition of $1.5 \mathrm{ml}$ of APTES (3-aminopropyl) triethoxyline $99 \%$ with a syringe; (3) stir during $10 \mathrm{~min}$; (4) pour $1.25 \mathrm{ml}$ of DI in a separate vial to which $19.81 \mathrm{~g}$ $(0.1 \mathrm{M})$ of $(+)$-Sodium L-ascorbate $\geq 98 \%$ (SA) are added; (5) stir during $10 \mathrm{~min}$; (6) the second vial is poured into the first one, while agitation is applied; (7) agitation continues during $30 \mathrm{~min}$; (8) samples are settled during $24 \mathrm{~h}$; (9) uncapped $5 \mathrm{ml}$ vials containing $1 \mathrm{ml}$ of each solution are placed in a desiccator during $24 \mathrm{~h}$; (10) sediments are recollected and incorporated, or mixed and then incorporated, to the PMMA (495 PMMA A2 from Microchem) to form the DSPL solution.

\section{Synthesis of CdTe quantum dots}

CdTe quantum dots were obtained using a chemical synthesis approach as described in [12]. The used process is as follows: (1) a solution of $2 \mathrm{~g}$ of $\mathrm{NaOH}$ in $50 \mathrm{ml}$ of DI is prepared and set aside; (2) in a $100 \mathrm{ml}$ flask, $0.053 \mathrm{~g}$ of cadmium acetate dihydrate $\left(\mathrm{Cd}\left(\mathrm{CH}_{3} \mathrm{COO}\right)_{2} \cdot 2 \mathrm{H}_{2} \mathrm{O}, 99.5 \%\right)$ is diluted in $50 \mathrm{ml}$ of DI and stirred for $5 \mathrm{~min}$; (3) $18 \mu \mathrm{l}$ of thioglycolic acid (TGA, 90\%) is added to the flask; (4) the solution $\mathrm{pH}$ is adjusted by dropwise adding the $\mathrm{NaOH}$ dispersion while agitating until a $\mathrm{pH}$ value between 10.5 and 11 is reached; (5) additional stirring is done during $5 \mathrm{~min}$; (6) in a separate flask, $0.0101 \mathrm{~g}$ of potassium tellurite $\left(\mathrm{K}_{2} \mathrm{TeO}_{3}\right.$, $95 \%$ ) is diluted in $50 \mathrm{ml}$ of water; (7) stirring is kept during $5 \mathrm{~min}$; (8) the potassium tellurite solution is added to the TGA solution; (9) stirring is done during $5 \mathrm{~min}$; (10) $0.08 \mathrm{~g}$ of sodium borohydride $\left(\mathrm{NaBH}_{4}, 99 \%\right)$ is added; (11) the mix is transferred to a single-neck, round-bottom flask attached to a Liebig condenser; (12) the flask is placed in an oil bath kept at $100{ }^{\circ} \mathrm{C}$ and the solution is refluxed during the desired synthesis time; based on a previous work [13], refluxing times of 8 and $12 \mathrm{~h}$ were chosen for this project. At the end of the refluxing time, the QDs' solutions were left at rest enough time, approximately $3 \mathrm{~h}$, to reach room temperature. Then, a volumetric relation of 1:1 of CdTe QDs solution and acetone (acetone HPLC, $\geq 99.9 \%$ Sigma-Aldrich) was poured in a plastic tube that was centrifuged for $10 \mathrm{~min}$ at $10,000 \mathrm{rpm}$. Once the residual liquid part was removed, the solids were dispersed again but this time with $1 \mathrm{ml}$ of DI and the solution was put in an ultrasonic bath (Branson 5510) for 
$5 \mathrm{~min}$. Next, $1 \mathrm{ml}$ of acetone was added and the solution was centrifuged for $10 \mathrm{~min}$ at $10,000 \mathrm{rpm}$. This procedure was repeated for three times after removing the remaining liquid part at the end of the ultrasonic bath and centrifugation.

\section{Si and CdTe QDs' blending}

Mixtures of Silicon and Cadmium telluride quantum dots were realized using four proportions, that is, $x \mathrm{Si}: y \mathrm{CdTe}-8 \mathrm{~h}$ and $x \mathrm{Si}: y \mathrm{CdTe}-12 \mathrm{~h}$ where the $x: y$ proportions corresponded to the volume ratios of $2: 1,1: 1,1: 2$, and $1: 3$ for both mixture types. Given that the 1:2 proportions showed better PL response than the one observed for the $2: 1$ case, it was decided to incorporate the $1: 3$ ratio and drop the $3: 1$ case. All blends were homogenized by a $15 \mathrm{~min}$ sonication step.

The QDs of Si, CdTe, or their mixtures, one at a time, were individually dispersed in $1 \mathrm{ml}$ of PMMA. In each case, four dispersions were realized in PMMA corresponding to individual QDs volumes of $0.5 \mathrm{ml}, 1.0 \mathrm{ml}, 1.5 \mathrm{ml}$, or $2.0 \mathrm{ml}$. Therefore, a total of 44 different coating samples were fabricated, 12 for the individual QDs and 16 for each one of the two mixtures according to all the possible combinations of the volume ratios $x: y$ and the proportions QDs:PMMA. Six previously characterized solar cells (bare cells) were used with each of these dispersions where, in each case, the dispersion was applied by spin-coating according to the parameters: $300 \mu \mathrm{l}$ dose, $4000 \mathrm{rpm}, 50 \mathrm{~s}$, and approximately $66 \mathrm{~nm}$ of layer thickness. Commercially available polycrystalline solar cells (Eco-worthy Company) were used (ARC finish, $52 \mathrm{~mm} \times 38 \mathrm{~mm}, 200 \mu \mathrm{m}$ thick). After the spin-coating process, the substrate was placed on a plate at $180^{\circ} \mathrm{C}$ to evaporate the solvents and to fix the thin films.

\section{Size and band-gap calculations}

Material bandgaps were determined using Tauc's graphical procedure [14] based on the following expression:

$\alpha=\frac{A}{E_{\mathrm{ph}}}\left(E_{\mathrm{ph}}-E_{\mathrm{g}}\right)^{m}$,

where $\alpha$ is the QDs absorption coefficient, $E_{\mathrm{ph}}$ is the incident photon energy, $E_{\mathrm{g}}$ is the bandgap, $A$ is a constant called the band tailing parameter, and $m$ is a parameter related to the nature of the exciton optical transition, which can only take the values of $0.5,2,3 / 2$, and 3 corresponding to allowed direct, allowed indirect, forbidden direct, and forbidden indirect transitions, respectively. The values of the diameters were calculated using the Brus equation [15]:

$E_{\mathrm{QD}}=E_{\mathrm{g}}^{\mathrm{bulk}}+\frac{\hbar^{2} \pi^{2}}{2 r^{2}}\left(\frac{1}{m_{e} * m_{0}}+\frac{1}{m_{h} * m_{0}}\right)-\frac{1.8 e^{2}}{4 \pi \epsilon \epsilon_{0} r}$, where $E_{\mathrm{QD}}$ is the band-gap energy of the quantum dot, $E_{\mathrm{g}}^{\text {bulk }}$ is the band-gap energy of the bulk semiconductor, $\hbar$ is the Planck constant, $m_{e}^{*}$ and $m_{h}^{*}$ are the effective masses of electrons and holes in a particular material, $m_{0}$ is the electron mass at rest, and $r$ is the radius of the quantum dot.

\section{Results and discussion}

\section{Size and band-gap results}

The estimated energy gaps and diameter values for the $\mathrm{Si}$ and CdTe QDs, along with their respective $m$ exponent, are given in Table 1 . Note that an allowed direct interband optical transition type was detected for the Si QDs case in contrast to the well-established indirect nature for the bulk material.

\section{QD optical characterization}

The UV-Vis absorption and the photoluminescence of the synthetized quantum dots solutions were recorded using an Ocean Optics Flame-S-UV-Vis spectrometer; for the emission spectra, an excitation wavelength of $380 \mathrm{~nm}$ was employed. The corresponding absorption and photoluminescent spectra for the individual and mixed QDs are shown in Fig. 3. Figure $3 a$ and $b$ shows the registered absorption and emission spectra, whereas Fig. $3 \mathrm{c}$ and d shows the corresponding normalized graphs of the emission spectra of the mixtures; the normalized spectra are to better evaluate the influence of the mixtures on the shift of the emission spectra. In fact, the intensity of the respective QDs peaks varied when modifying the volumetric content of each constituent in the dispersions (as shown in Fig. 3c, d). In addition, in both cases, the characteristic photoluminescent peaks of the $\mathrm{Si}$ and CdTe QDs were observed to exhibit a relatively small, but not negligible, shift as the mixture evolved from a Si-rich condition to a CdTe-rich dispersion. Specifically, in the case of the QDS dispersions involving Si and CdTe QDs with a synthesis time of $8 \mathrm{~h}$ (see Fig. 3c and Table 2), the Si peak was generally observed to be blue-shifted, whereas the CdTe

Table 1 Energy bandgap, particle diameter, and optical transition parameter value for QDs

\begin{tabular}{lllll}
\hline \multirow{2}{*}{ Parameter } & $E_{\mathrm{g}}(\mathrm{eV})$ & 2.47 & 2.28 & 2.15 \\
\cline { 3 - 5 } & $\mathrm{Si}$ & CdTe-8 h & CdTe-12 h \\
\cline { 3 - 5 } & QDs D $(\mathrm{nm})$ & 2.79 & 3.21 & 3.46 \\
& $m$ & 0.5 & 0.5 & 0.5 \\
\hline
\end{tabular}




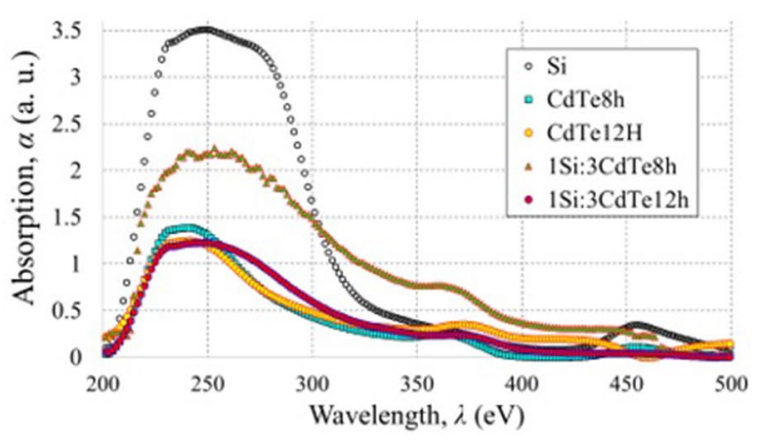

(a)

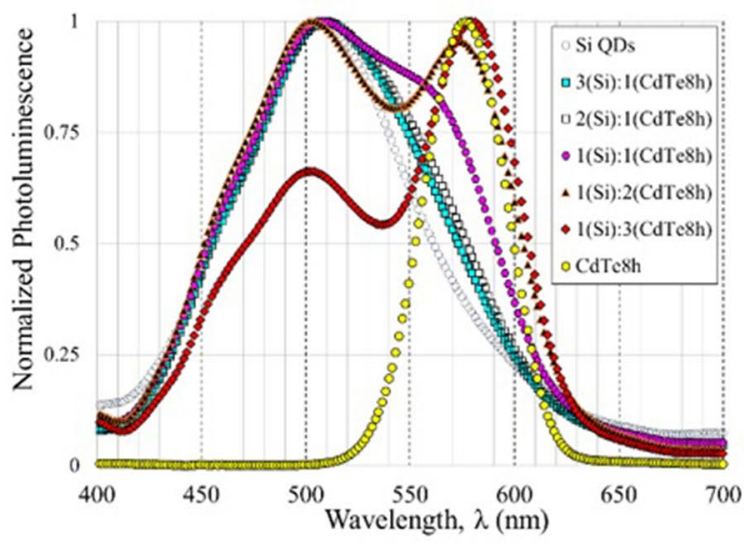

(c)

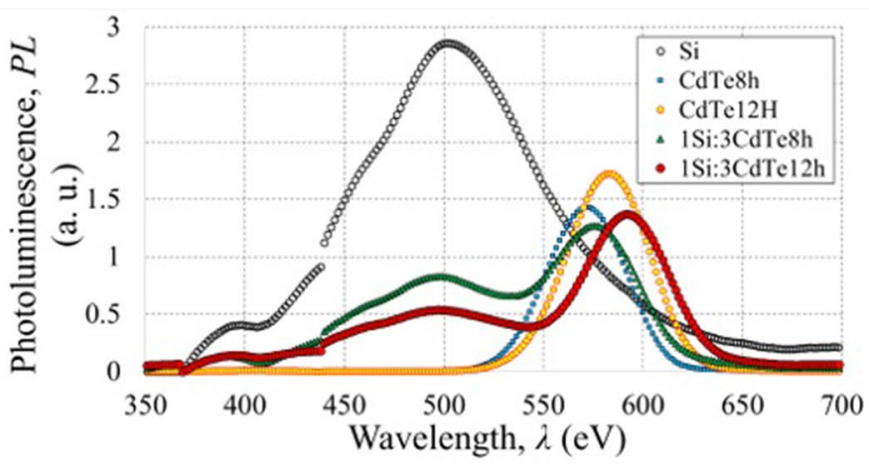

(b)

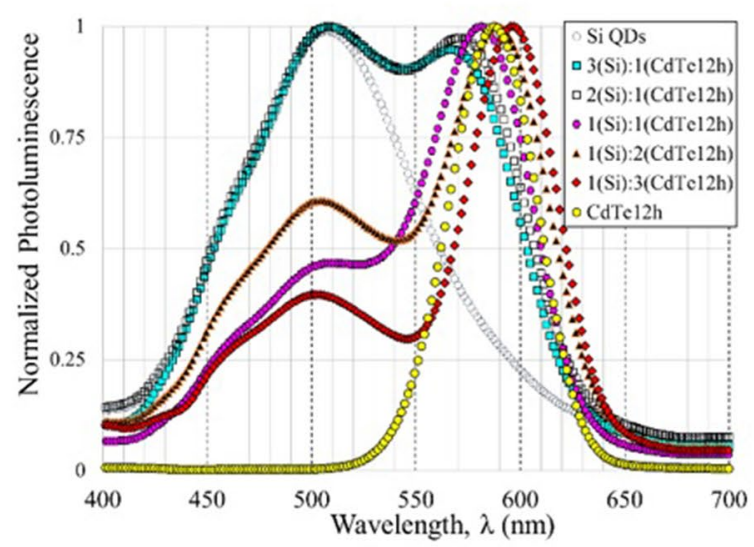

(d)

Fig. 3 Absorption and photoluminescent emission spectra of Si and CdTe QDs dispersions

Table 2 Shift observed in the characteristic PL peaks in $\mathrm{Si}$ and CdTe-8 h QD heterogeneous dispersions

\begin{tabular}{lll}
\hline Si:CdTe QD ratio & Si peak shift $(\mathrm{nm})$ & $\begin{array}{l}\text { CdTe-8 } \mathrm{h} \\
\text { peak shift } \\
(\mathrm{nm})\end{array}$ \\
\hline $2: 1$ & -4.3 & -8.1 \\
$1: 1$ & -3.8 & -4.1 \\
$1: 2$ & -4.3 & +4.9 \\
$1: 3$ & -5.3 & +5.9 \\
\hline
\end{tabular}

peak migrated from a blue-shift of $\sim 8.1 \mathrm{~nm}$ in a Si-rich dispersion to a red-shift of $\sim 5.9 \mathrm{~nm}$ in a CdTe-rich condition.

In view of the wavelength-dependent absorption probability displayed in Fig. 1, the prospective control of the red-shift of CdTe QD PL peak is of interest because of its anticipated influence on solar cell performance. The quantum dot interaction previously described also has a dependence on the size of the nanoparticles involved. Specifically, dispersions comprised of CdTe QDs with a synthesis time of $12 \mathrm{~h}$ in combination with the aforementioned
Table 3 Shift observed in the characteristic PL peaks in Si and CdTe$12 \mathrm{~h}$ QD heterogeneous dispersions

\begin{tabular}{lll}
\hline Si:CdTe QD ratio & Si peak shift $(\mathrm{nm})$ & $\begin{array}{l}\text { CdTe-12 h } \\
\text { peak shift } \\
(\mathrm{nm})\end{array}$ \\
\hline $2: 1$ & -0.3 & -5.8 \\
$1: 1$ & -1.3 & -3.8 \\
$1: 2$ & -1.3 & +5.2 \\
$1: 3$ & -5.3 & +10.2 \\
\hline
\end{tabular}

Si QDs exhibited a similar behavior as that observed with smaller CdTe QDs (see Fig. 3d and Table 3) where the Si peak was also observed to be blue-shifted; however, the CdTe- $12 \mathrm{~h}$ peak migrated from a blue-shift of $\sim 5.8 \mathrm{~nm}$ in a Si-rich dispersion to a red-shift of $\sim 10.2 \mathrm{~nm}$ in a CdTerich condition.

The observed effects are thought to be due to quantum dot physical adsorption leading to surface energy variations. However, the computational modeling of the observed effects has not been performed to date. 


\section{Silicon solar cell coating and characterization with QDs}

Based on the previous observations, the $1 \mathrm{Si}: 3 \mathrm{CdTe}-12 \mathrm{~h}$ mixture was selected to incorporate on solar cells, since it exhibited the largest red-shift, and the CdTe-12 h has the lowest band-gap energy, or the highest size, as can be seen in Table 1. Besides, its emission spectrum is located at longer wavelength regarding the $\mathrm{Si}$ and $\mathrm{CdTe}-8 \mathrm{~h}$ QDs. Sonication was employed to disperse the blend in polymethylmethacrylate (495 PMMA Microchem) prior to coating the window side of a set of polycrystalline Si solar cells using the spin-coating method [16]. There were six solar cells tested for each different condition considered in this study; that is, test of the bare solar cells and then test of the coated solar cells, considering the individual volume ratios with each individual QDs and each mixture.

Each set of polycrystalline solar cells was measured without and with the incorporation of the aforementioned downshifting QDs using an AM1.5 solar simulator (Oriel Sol2A). The collected values of open-circuit voltage $\left(V_{\mathrm{oc}}\right)$, shortcircuit current $\left(J_{\mathrm{sc}}\right)$, and fill factor $(\mathrm{FF})$ were recorded (see Table 4) and employed to calculate the power conversion efficiency (PCE) of the devices tested, which increased from 14.6 to $14.78 \%$ in the best case when the down-converting film was incorporated to the solar cells.

For a comparative analysis, the external quantum efficiency (EQE) was registered for the bare and after coated solar cells; the $\Delta$ s reported in Table 4 were determined with the calculated values, not with the rounded ones. According to both results, the Si QDs have presented the smallest difference $(0.39 \%$ as shown in Table 4$)$ between prior and after the application of the thin film of PMMA/Si QDs, see Fig. 4a. On the other hand, the EQE spectrum for the solar cells without and with the thin film of PMMA/CdTe- $12 \mathrm{~h}$ QDs shows a better benefit than the cells incorporating the PMMA/Si QDs layer (0.48\% as reported in Table 4$)$, see
Fig. 4b. Nevertheless, the benefit for the Si QDs is within the uncertainty, so it practically remained unchanged, as can be seen in Table 4 for the rounded values and their corresponding uncertainty. The solar cells with the PMMA/CdTe- $8 \mathrm{~h}$, on the other hand, gave the best increase in efficiency of the individual QDs of this study $(0.83 \%$ according to results in Table 4), though the mixture of PMMA/Si:CdTe-12 h was the best of the mixtures ( $1.53 \%$ as given in Table 4$)$. Specifically, these results correspond to the volume ratios of $1 \mathrm{ml} / 2 \mathrm{ml}$ for PMMA/(individual QDs: Si, CdTe- $8 \mathrm{~h}$, or CdTe-12 h) and $1 \mathrm{ml} / 1.5 \mathrm{ml}$ for PMMA/(Si:CdTe-12 h).

The smoothed EQEs for bare and coated cells are shown in Fig. 4. In all cases, there is a better use of high-energy photons by the use of the QDs, as can be appreciated on the left side of the EQEs and corresponding to the downshifting effect of the QDs used in this work. In addition, the transition between both types of EQEs, with and without QDs, for the high-energy photons is realized according to the photoluminescence response of the QDs. As shown by the EQEs of the Si:CdTe-12 h QDs mixture in Fig. 4d, there is a greater contribution of the more energetic photons and also the transition of both EQEs occurs at a longer wavelength, this as a combined result of the down-shifting and the red-shift effects (see Fig. 3 and Table 3). Therefore, it is reasonably to get a greater efficiency, calculated in this case as an increment of $1.53 \%$.

\section{Conclusions}

Our results indicate that it is possible to obtain a PCE increase of $1.53 \%$ in Si solar cells using a QDs mixture of 1Si:3CdTe12 h; individually, none of the QDs considered in our study showed a better efficiency boost. This result is mostly due to the observed red-shift of the DSPL of mixtures which produce photons with increased EQE in Si. Therefore, the ability to modify the down-shifting characteristics of
Table 4 Solar cell characterization

\begin{tabular}{lllccl}
\hline Samples & & $V_{\text {oc }}(\mathrm{mV})$ & $J_{\text {sc }}\left(\mathrm{mA} / \mathrm{cm}^{2}\right)$ & FF $(\%)$ & PCE $(\%)$ \\
\hline Si QDs & Bare cells & 612.1 & 34.90 & 69.2 & $14.8 \pm 0.3$ \\
& Si QDs & 612.2 & 34.88 & 69.5 & $14.8 \pm 0.4$ \\
& $\% \Delta$ of Means & 0.016 & -0.06 & 0.43 & 0.39 \\
CdTe8 QDs & Bare cells & 604.0 & 34.39 & 70.0 & $14.54 \pm 0.36$ \\
& CdTe8 QDs & 604.6 & 34.56 & 70.2 & $14.66 \pm 0.45$ \\
CdTe12 QDs & $\% \Delta$ of Means & 0.09 & 0.51 & 0.23 & 0.83 \\
& Bare cells & 613.0 & 34.6 & 68.9 & $14.62 \pm 0.06$ \\
& CdTe12 QDs & 615.4 & 34.27 & 69.3 & $14.68 \pm 0.11$ \\
1Si:3CdTe12 QDs & $\% \Delta$ of Means & 0.39 & -0.95 & 0.58 & 0.48 \\
& Bare cells & 615.5 & 34.38 & 68.8 & $14.6 \pm 0.1$ \\
& 1Si:3CdTe12 QDs & 617.9 & 34.72 & 68.9 & $14.78 \pm 0.06$ \\
& $\% \Delta$ of Means & 0.39 & 0.99 & 0.15 & 1.53 \\
\hline
\end{tabular}




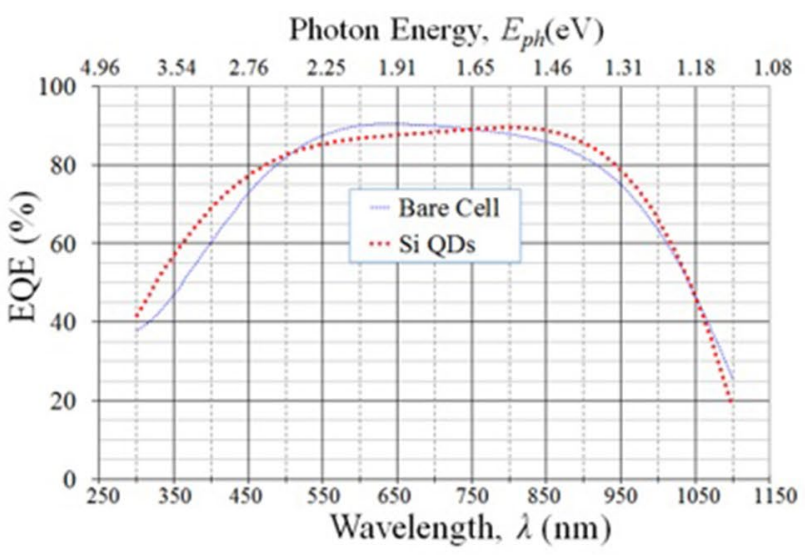

(a)

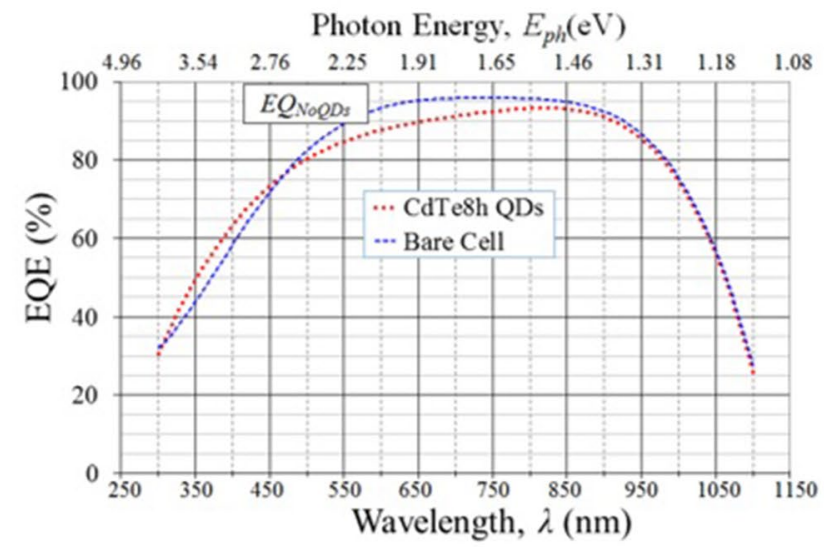

(c)

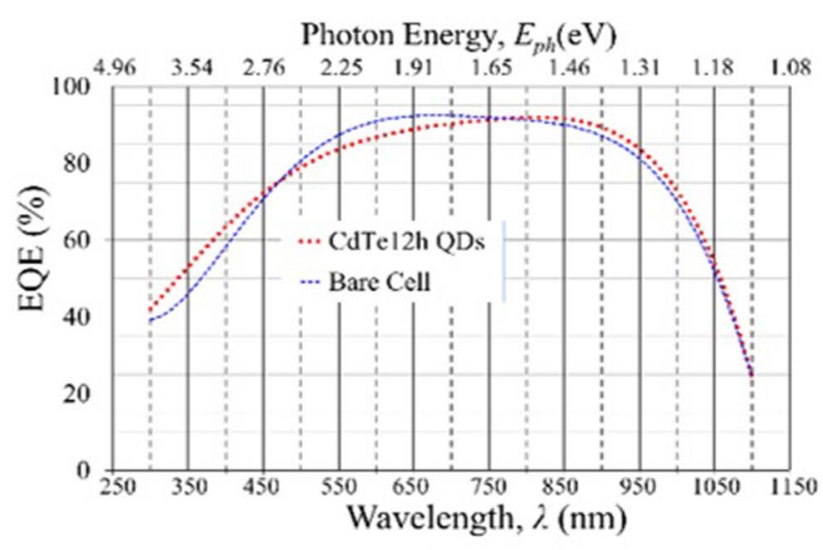

(b)

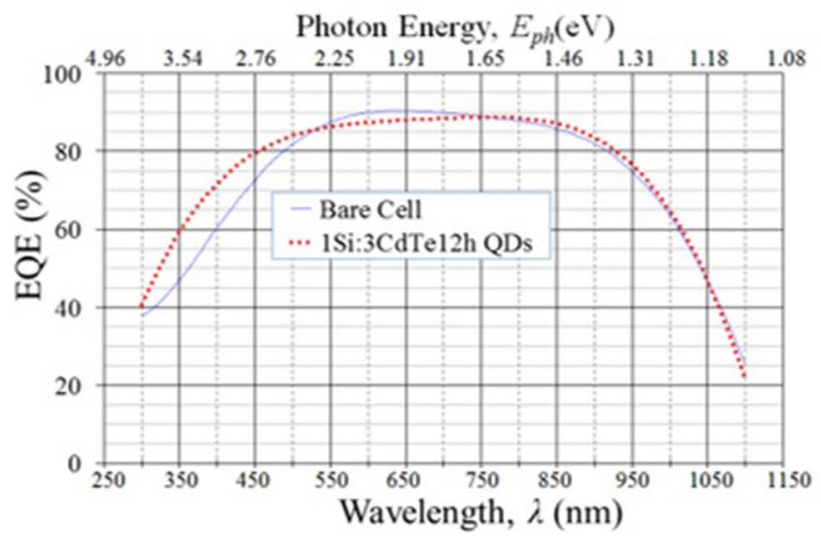

(d)

Fig. 4 EQE spectra of solar cells with and without Si and/or CdTe-12 h QDs

quantum dots by employing heterogeneous mixtures could prove a valuable tool to provide cost reduction of photovoltaic structures for power generation.

Acknowledgements The authors would like to acknowledge the National Science Foundation Grant No. ECCS 1650571 U.S. Army Research office (Grant W911NF-13-1-0110), CONACYT, the Department of Physics and Astronomy, University of Texas at San Antonio, and the Department of Projects Engineering-CUCEI, University of Guadalajara, México, for the support provided for this project.

Open Access This article is distributed under the terms of the Creative Commons Attribution 4.0 International License (http://creativeco mmons.org/licenses/by/4.0/), which permits unrestricted use, distribution, and reproduction in any medium, provided you give appropriate credit to the original author(s) and the source, provide a link to the Creative Commons license, and indicate if changes were made.

\section{References}

1. Hirst, L.C., Ekins-Daukenes, N.J.: Fundamental losses in solar cells. Prog. Photovolt. 19(3), 286-293 (2011)

2. Nilofar, A., Kamaruzzaman, S., Shideh, A., Kasra, S., Alghoul, M., Omidreza, S., Saleem, H.Z.: A review on the role of materials science in solar cells. Renew. Sustain. Energy Rev. 16, 5834-5847 (2012)

3. Lopez-Delgado, R., Higareda-valenzuela, H., Zazueta-Raydnaud, A., Pelayo, J.E., Ayon, A.: Solar cell efficiency improvement employing down-shifting silicon quantum dots. Microsyst. Tecnol. 24(1), 495-502 (2017)

4. Rowan, B.C., Wilson, L.R., Richards, B.S.: Advance materials concepts for luminiscent solar concentrator. Sel. Top. Quant. Electron. 14(5), 1312-1322 (2008)

5. Krammer, I.J., Sargent, E.H.: The architecture of colloidal quantum dots solar cells: materials to device. Chem. Rev. 114, 863-882 (2014)

6. Juanita, K.: Improved solar cell efficiency through the use of an additive nanostructure-based optical downshifter. Spectrawatt Inc., Hillsboro (2011)

7. Klampaftis, E., Ross, D., Mclntosh, K.R., Bryce, S.R.: Enhancing the performance of solar cells via luminescent down-shifting of 
incident spectrum. Sol. Energy Mater. Sol. Cells 93, 1182-1194 (2009)

8. Pelayo, E., Zazueta, A., Lopez, R., Saucedo, E., Ruelas, R., Ayon, A.: Silicon solar cell efficiency improvement employing the photoluminescent, down shifting effects of carbon and CdTe quantum dots. Mater. Renew. Sustain. Energy. 5(5), 1-7 (2016)

9. Du, X.W., Fu, Y.S., Su, J., Han, X., Liu, J.: Complete UV emission of $\mathrm{ZnO}$ nanoparticles in a PMMA matrix. Semicond. Sci. Technol. 21, 1202-1206 (2006)

10. Lopez-Delgado, R., Higareda-Valenzuela, H., Zazueta-Raynaud, A., Ramos, A., Pelayo, J.E., Ayon, A.: Enhancing the power conversion efficiency of solar cells employing down-shifting silicon quantum dots. J. Phys. 773, 1-4 (2016)

11. Wang, J., Ye, D.X., Liang, G.-H., Chang, J., Kong, J.-L., Ji-Yao, C.: One-step synthesis of water dispersible silicon nanoparticles and their use in fluorescence lifetime imaging of living cells. J. Mater. Chem. B. 2, 4338-4345 (2014)

12. Wu, S., Dou, J., Zhang, J., Zhang, S.: A simple and economical one-pot method to synthesize high-quality water soluble CdTe QDs. Mater. Chem. 22, 14573-14578 (2012)
13. Tronco-Jurado, U., Saucedo Flores, E., Ruelas, R., López, R., Alvarez Ramos, M.E., Ayón, A.A.: Synergistic effects of nanotexturization and down shifting CdTe quantum dots in solar cell performance. Microsyst. Technol. 23, 3945-3953 (2017)

14. Tauc, J.: Optical properties and electronic structure of amorphous Ge and Si. Mater. Res. Bull. 3(1), 37-46 (1968)

15. Brus, L.: Electronic wave functions in semiconductor clusters: experiment and theory. J. Phys. Chem. 90(2), 2555-2560 (1986)

16. Mihi, A., Ocaña, M., Miguez, H.: Oriented colloidal-crystal thin films by spin-coating microspheres dispersed in volatile media. Adv. Mater. 18, 2244-2249 (2006)

Publisher's Note Springer Nature remains neutral with regard to jurisdictional claims in published maps and institutional affiliations. 